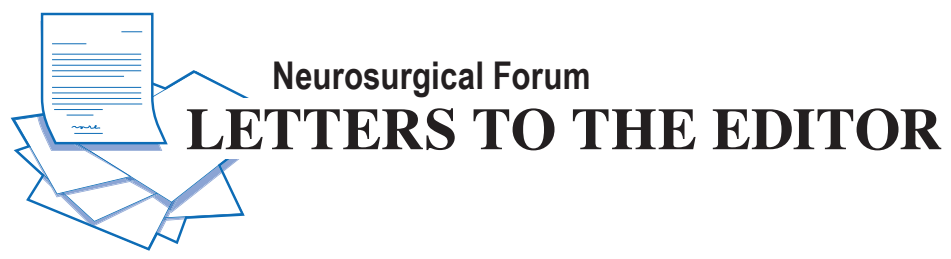

\section{Reversal of low prealbumin with oral branched-chain amino acids: a simple solution to an expensive problem}

TO THE EDITOR: We read with interest the article by Salvetti et al. ${ }^{4}$ (Salvetti DJ, Tempel ZJ, Goldschmidt E, et al: Low preoperative serum prealbumin levels and the postoperative surgical site infection risk in elective spine surgery: a consecutive series. J Neurosurg Spine 29:549552 , November 2018). This study provided much needed awareness on the importance of nutrition to overall outcomes. We recently commented on the need for revisions to our neurosurgical organizations' (AANS/CNS) guidelines for the treatment of severe traumatic brain injury on the basis of the strong literature support for early treatment with branched-chain amino acids (BCAAs). ${ }^{1}$ Guan et al. recently published a similar study comparing outcomes on patients with low prealbumin levels to patients with normal preoperative levels who underwent spine surgery. ${ }^{2}$ The study found a significantly higher rate of complications in patients with abnormally low prealbumin levels. ${ }^{2}$

Nutritional status continues to be one of the top risk factors for complications during hospitalization and in the postoperative period. ${ }^{3}$ Ruiz et al. followed 800 malnourished hospitalized patients and demonstrated increased length of hospitalization, increased mortality rate, as well as increased hospital costs. ${ }^{3}$ The study concluded that improving diagnosis and treatment of malnutrition would improve patient outcomes and reduce healthcare costs. ${ }^{3}$ In this era of specialized medicine, most neurosurgical patients are co-managed with hospitalists, trauma surgeons, etc., and we are not necessarily focused on prealbumin levels, nor are we informed how to treat low prealbumin levels in the perioperative period. We recently completed a study on 25 postoperative neurosurgical patients who were undergoing inpatient rehabilitation and had low prealbumin levels. The study was specifically designed to identify patients with low prealbumin levels and attempt to increase the prealbumin levels with an oral BCAA supplement. The reason for the study was to demonstrate how easily low prealbumin levels can be reversed without the risks and costs associated with total parenteral nutrition or intravenous supplements. ${ }^{5}$ We found that after 7 days of oral BCAA supplementation, all 25 patients' prealbumin levels were elevated back to within normal limits $(\mathrm{p}<$ 0.05). We now recommend BCAA supplementation along with other basic nutritional advice to all our patients. The recent changes among insurance companies and Medicare to reduce medical expenses through denying approval for certain medications, procedures, and surgeries and furthermore penalizing the hospitals for postoperative complications that occur within 30 days of surgery has brought an increasing awareness among hospitals and physicians on how to provide the best preventive medicine while reducing risks. We congratulate Salvetti et al. on their contribution to the neurosurgical literature and also thank the JNS Publishing Group for publishing an article that brings awareness to our neurosurgical community on optimizing nutritional status to improve surgical outcomes. ${ }^{4}$

Rob Dickerman, DO, PhD

Presbyterian Hospital of Plano, University of North Texas Health Science Center, Plano, TX

Julie Williamson, BS

North Texas Brain and Spine Institute, Plano, TX

Matthew Bennett, MD

United Health Services, Johnson City, NY

\section{References}

1. Dickerman R, Reynolds A, Williamson J, Winters K: Guidelines for the Management of Severe Traumatic Brain Injury, Fourth Edition. Neurosurgery 81:E50, 2017 (Letter)

2. Guan J, Holland CM, Ravindra VM, Bisson EF: Perioperative malnutrition and its relationship to length of stay and complications in patients undergoing surgery for cervical myelopathy. Surg Neurol Int 8:307, 2017

3. Ruiz AJ, Buitrago G, Rodriguez N, Gómez G, Sulo S, Gómez $\mathrm{C}$, et al: Clinical and economic outcomes associated with malnutrition in hospitalized patients. Clin Nutr [epub ahead of print], 2018

4. Salvetti DJ, Tempel ZJ, Goldschmidt E, Colwell NA, Angriman F, Panczykowski DM, et al: Low preoperative serum prealbumin levels and the postoperative surgical site infection risk in elective spine surgery: a consecutive series. J Neurosurg Spine 29:549-552, 2018

5. Wang XY, Li N, Gu J, Li WQ, Li JS: The effects of the formula of amino acids enriched BCAA on nutritional support in traumatic patients. World J Gastroenterol 15:599-602, 2003 


\section{Disclosures}

The authors report no conflict of interest.

\section{Correspondence}

Rob Dickerman:drrdd@yahoo.com.

INCLUDE WHEN CITING

Published online October 26, 2018; DOI: 10.3171/2018.8.SPINE18925.

\section{Response}

We thank Dr. Dickerman and colleagues for their insight regarding the correction of nutritional deficiencies and their kind words in reference to our recent publication. Their additional references reinforce the need for continued attention to this subject. We also commend their efforts on researching a solution to improving a patient's nutritional status with supplementation of BCAAs. Improving a patient's nutritional status remains at the forefront in altering surgical risk profiles.

As the field of neurosurgery continues to investigate the effects of nutrition on outcomes, there is a critical concept that we would like to reinforce: prealbumin is involved in the acute phase response and therefore lowered in states of inflammation., ${ }^{1,2}$ Consequently, prealbumin levels measured in the inpatient, postacute, or postoperative setting must be interpreted with caution. In the setting of a known state of illness, it is not clear to what extent we are measuring a response to inflammation versus a true nutritional deficiency. In our 2015 study, prealbumin was measured in 83 patients at the time of presentation with a postoperative surgical site infection, and $82(99 \%)$ of these patients demonstrated a low prealbumin level. ${ }^{3}$ Because the measurement was taken at the time the infection was identified, the significance of whether a low prealbumin level indicated inflammation or malnutrition is difficult to differentiate. The design of our current study attempted to avoid this confounding factor by measuring prealbumin in a state of relative health during the preoperative workup phase. Even this method is imperfect because measurement could be confounded by other systemic diseases promoting inflammation, but it is presumably a better reflection of nutritional status than measurement during a known inflammatory reaction. Simultaneously measuring other inflammatory markers could potentially be useful to help differentiate general inflammation from a state of an isolated low prealbumin level and potential nutritional deficiency.

The question of prealbumin levels being an accurate reflection of nutritional intake will be answered by whether we can influence the risk of complications and outcomes through nutritional supplementation. The ideal next step is a high-quality, placebo-controlled prospective study with adequate power designed with the limitations of prealbumin measurement in mind. In an era of value-based care, proving that this is a cost-effective strategy will also be critical.

David J. Salvetti, MD

Adam S. Kanter, MD David O. Okonkwo, MD, PhD University of Pittsburgh Medical Center, Pittsburgh, PA

\section{References}

1. Dellière $\mathrm{S}$, Cynober L: Is transthyretin a good marker of nutritional status? Clin Nutr 36:364-370, 2017

2. Lee JL, Oh ES, Lee RW, Finucane TE: Serum albumin and prealbumin in calorically restricted, non-diseased individuals: a systematic review. Am J Med 128:1023.e1-1023.e22, 2015

3. Tempel Z, Grandhi R, Maserati M, Panczykowski D, Ochoa $\mathrm{J}$, Russavage J, et al: Prealbumin as a serum biomarker of impaired perioperative nutritional status and risk for surgical site infection after spine surgery. J Neurol Surg A Cent Eur Neurosurg 76:139-143, 2015

INCLUDE WHEN CITING

Published online October 26, 2018; DOI: 10.3171/2018.9.SPINE18956.

CAANS 2019, except where prohibited by US copyright law

\section{Epidural blood patch in superficial siderosis caused by spontaneous intracranial hypotension}

TO THE EDITOR: We note with interest the article by Arishima et al. '. (Arishima H, Higashino Y, Yamada S, et al: Spinal endoscopy combined with selective CT myelography for dural closure of the spinal dural defect with superficial siderosis: technical note. J Neurosurg Spine 28:96-102, January 2018). The authors described an innovative method to detect tiny dural holes in two patients suffering from superficial siderosis (SS) and CSF leakage. They performed spinal endoscopy using a coronary angioscope system and selective CT myelography by utilizing a spinal drainage tube. Under fluoroscopy, they introduced the coronary angioscope into the spinal subarachnoid space, a procedure comparable to the spinal drainage, and slowly advanced the scope to the cervical spine. The angioscope clearly showed each small dural hole and injured arachnoid membrane. One week later, the spinal drainage tube was inserted, and the tip of the drainage tube was located just below the level of the dural defect found by the spinal endoscopic examination. This selective CT myelography clarified the location of the dural defect. During surgery, the small dural hole could be easily located and safely sutured. The two patients reported on had a history of surgery for bilateral chronic subdural hematoma (CSDH) 15 and 20 years previously. They continued to have headaches even after the surgery. We noticed the authors did not describe the headache features at the time of CSDH evacuation. We think the patients probably suffered at onset with an orthostatic headache. Bilateral subdural hematomas could be a complication of spontaneous intracranial hypotension (SIH) that was misdiagnosed at the time of CSDH evacuation. The authors did not report whether, before the hematomas were drained, the patients had undergone brain MRI, which could have identified typical SIH neuroradiological features such as symmetrical and linear diffuse pachymeningeal enhancement that would have allowed an SIH diagnosis.

The persistence of the headache, likely orthostatic in nature, after hematoma drainage would further suggest an 
SIH diagnosis in these 2 patients. On the other hand, the low CSF pressure finding at the time of lumbar puncture would confirm the hypothesis that these 2 patients initially suffered from SIH. Furthermore, both patients had, as evidenced on spinal T2-weighted MRI, a longitudinal epidural fluid collection ventral to the spinal cord (from $\mathrm{C} 2$ to T12). These data confirm the presence of a spinal fluid fistula in the spinal cord segments between C2 and T12. In this regard, it is thought that many patients with SIH have a longitudinal epidural fluid collection ventral to the spinal cord. In our series of over 400 patients with SIH, observed from 1992 to 2018, a spinal ventral epidural fluid collection was found in about $35 \%$ of patients. Of approximately 400 patients with SIH observed, about 300 (75\%) were treated with autologous lumbar epidural blood patch (EBP) and achieved good outcomes. ${ }^{3,4}$ Patients with a longitudinal ventral epidural fluid collection in thoracic and cervical spinal segments were also treated using EBP and had good outcomes. ${ }^{2}$

Based on our experience with a large cohort of SIH patients, in these 2 cases described by Arishima et al., ${ }^{1}$ we would have first suggested a less invasive treatment with autologous lumbar EBP, keeping the innovative spinal surgical procedure performed by the authors as secondchoice treatment, just in case the autologous lumbar EBP failed.

Enrico Ferrante, MD

Michele Trimboli, MD

AOR San Carlo, Potenza, Italy

\section{References}

1. Arishima H, Higashino Y, Yamada S, Akazawa A, Arai H, Tsunetoshi K, et al: Spinal endoscopy combined with selective CT myelography for dural closure of the spinal dural defect with superficial siderosis: technical note. J Neurosurg Spine 28:96-102, 2018

2. Ferrante E, Arpino I, Citterio A: Is it a rational choice to treat with lumbar epidural blood patch headache caused by spontaneous cervical CSF leak? Cephalalgia 26:1245-1246, 2006

3. Ferrante E, Arpino I, Citterio A, Wetzl R, Savino A: Epidural blood patch in Trendelenburg position pre-medicated with acetazolamide to treat spontaneous intracranial hypotension. Eur J Neurol 17:715-719, 2010

4. Ferrante E, Rubino F, Arpino I, Beretta F, Citterio A, Pero G, et al: Treatment of orthostatic headache from spontaneous intracranial hypotension syndrome: single institutional experience of 326 cases. J Headache Pain 16 (Suppl 1):A125, 2015 (Abstract)

\section{Disclosures}

The authors report no conflict of interest.

\section{Correspondence}

Enrico Ferrante: enricoferrante@libero.it.

\section{INCLUDE WHEN CITING}

Published online October 26, 2018; DOI: 10.3171/2018.9.SPINE181146.

\section{Response}

We thank Drs. Ferrante and Trimboli for their comments and would like to take this opportunity to respond.

As Drs. Ferrante and Trimboli suggested, we also think that our patient had SIH due to a spinal dural defect and were misdiagnosed at the time of surgery for bilateral CSDH. The two patients in our article had a history of surgery for bilateral CSDH about 20 years previously, and they were treated at another hospital. As far as we know, they had neither a history of head trauma nor bleeding tendency before surgery for bilateral CSDH. The patient in case 1 underwent bed rest for a few weeks after removal of the CSDH. The patient did not remember any headache features. Regrettably, we could not get more information about their MRI or the operations performed with drainage.

A lumbar EBP may be useful and less invasive for SIH patients in whom there is a longitudinal epidural fluid collection dorsal to the spinal cord; however, we question its use when surely applied close to a ventral spinal dural defect in patients with SS. The purpose of our technical note was to accurately detect the location of dural defects that cannot be demonstrated by conventional imaging. We hope that the usefulness of a lumbar EBP for SS patients with $\mathrm{SIH}$ will be discussed in future.

Hidetaka Arishima, MD

University of Fukui, Japan

\section{INCLUDE WHEN CITING}

Published online October 26, 2018; DOI: 10.3171/2018.10.SPINE181194.

CAANS 2019, except where prohibited by US copyright law 